\title{
A behavioral medicine intervention for community-dwelling older adults with chronic musculoskeletal pain: protocol for a randomized controlled trial
}

\author{
This article was published in the following Dove Press journal: \\ Journal of Pain Research \\ 10 April 2017 \\ Number of times this article has been viewed
}

\section{Sara Cederbom' \\ Eva Denison ${ }^{2}$ \\ Astrid Bergland'}

'Department of Physiotherapy, Faculty of Health Sciences, Oslo and Akershus University College of Applied

Sciences, Oslo, Norway; ${ }^{2}$ Department of Physiotherapy, School of Health, Care and Social Welfare, Mälardalen University, Västerås, Sweden
Correspondence: Sara Cederbom Department of Physiotherapy, Faculty of Health Sciences, Oslo and Akershus University College of Applied Sciences, PO Box 4, St. Olavs Plass, N-0130 Oslo, Norway

Tel +4767236802

Email sara.cederbom@hioa.no
Background: Chronic musculoskeletal pain is a major health problem among older adults, particularly those who live alone and/or those who are dependent on formal care. Chronic pain is associated with mobility problems, falls, fear of falling, catastrophizing thoughts, and a lower quality of life. Research shows that physical therapy interventions based on behavioral medicine approaches are beneficial for middle-aged adults with chronic pain. However, there appears to be no previous randomized controlled trials (RCTs) based on this theoretical framework that have examined the effect on older adults with chronic musculoskeletal pain who live alone at home and are dependent on formal care to manage their everyday lives. The aim of the planned study is to evaluate the effect of an individually tailored integrated physical therapy intervention based on a behavioral medicine approach compared with the effect of standard care.

Methods/design: The planned study is an RCT that will include one intervention and one control group involving a total of 150 adults aged $\geq 75$ years with chronic musculoskeletal pain who live alone at home and are dependent on formal care to manage their everyday lives. The intervention will involve a 12 -week home-based individually tailored intervention that will be designed to enhance the participants' ability to perform everyday activities by improving physical function and reducing pain-related disability and beliefs. The control group will be given standard care, including general advice about physical activity. The participants will be assessed at baseline and at 3 and 6 months after baseline. The primary outcome will be painrelated disability and physical performance.

Discussion: The intervention, if effective, will have the potential to be the basis of the first evidence-based guidelines for physical therapists who work with older adults with chronic musculoskeletal pain.

Keywords: fall, fear of falling, independence, physical activity, self-efficacy, quality of life

\section{Background}

In Norway, as in other countries around the world, care is oriented toward home-based services, with support from the community. ${ }^{1-5}$ An important goal for the care of older people is to help them to maintain their ability to perform everyday activities, which is especially important for older community-dwelling adults who live with chronic pain. ${ }^{6,7}$ Everyday activities have been shown to be important for maintaining older individuals' independence and quality of life.$^{8,9}$ Loss of the ability to perform everyday activities has been shown to be strongly associated with institutionalization, increased caregiver burden, higher resource use, and risk of death. ${ }^{10,11}$ 
Elderly individuals who live alone seem to be more vulnerable to health issues than those who live with others. Several studies have shown that individuals who live alone have poorer health, a higher degree of pain, greater disability with regard to the instrumental activities of daily living (I-ADL), a greater degree of functional decline, more falls, and more activity limitations related to the fear of falling. Living alone is also associated with a higher degree of mental illness (including symptoms of depression), and individuals who live alone report that they have a decreased quality of life compared to those who do not live alone. ${ }^{12-16}$ The majority of people who depend on formal help to manage their everyday lives live alone. ${ }^{4}$

As mentioned earlier, chronic pain is a major health problem among the elderly, especially among those who live alone. The prevalence of chronic pain among communitydwelling older adults ranges from $45 \%$ to $80 \%$, and it is more common among women. The most common type of pain among older adults is chronic musculoskeletal pain. ${ }^{717}$

Chronic pain among older people is associated with multiple pain-related issues, such as mobility problems, fatigue, social isolation, sleep disturbance, psychological distress, depression, and a lower quality of life. ${ }^{7}$ In addition, a recently published study showed that older people with chronic musculoskeletal pain have a substantially greater risk of developing a disability and impaired mobility performance over time compared to older adults without chronic pain. ${ }^{18}$ Similar results have also been found among older people receiving home care. ${ }^{17}$

Living with chronic musculoskeletal pain is also associated with psychological distress, that is, fear of movement and pain catastrophizing, and these beliefs have a strong influence on the development and maintenance of painrelated disability. Only in recent years, there has been a focus in this field on older people. ${ }^{19-21}$ Cederbom et al ${ }^{22}$ carried out a study that focused on community-dwelling older women with chronic pain who lived alone and were dependent on formal care, and they found that pain catastrophizing had a negative impact on both pain-related disability and psychological well-being.

Another major issue among older people is falling and fear of falling. Individuals who have chronic pain have been reported to have a greater risk of falling and a greater risk of developing a fear of falling. ${ }^{23,24}$ Despite this, there are few interventional studies on improving physical performance to reduce the risk of falling or on reducing the fear of falling, and there are fewer still among community-dwelling older adults with chronic pain. ${ }^{23}$
Nevertheless, chronic pain among older people remains underassessed, underdiagnosed, and undertreated, despite its negative impact on physical and mental health, physical performance, and quality of life. ${ }^{25,26}$ Evidence-based guidelines for the treatment of chronic pain in community-dwelling older adults that focus on maintaining and improving physical independence, as well as improving general health and quality of life, are emerging for physical therapists who work with this population.

Interventions based on individually tailored integrated behavioral medicine approaches in physical therapy (BMPI) consider medical, physical, behavioral, cognitive, psychological, and social environmental factors in the analysis of the issues to be addressed and the subsequent treatment. These interventions have been shown to improve individuals' abilities to perform everyday activities and the level of physical activity among middle-aged patients with chronic pain..$^{27-29}$ However, research on the effects of this kind of intervention for older adults remains inadequate. The only study that the authors have identified is a recently published feasibility study by Cederbom et al that involved a BMPI for older women with chronic pain who lived alone and were dependent on formal care..$^{30}$ The study found that the intervention was feasible for use in the target population and that the intervention was able to facilitate both physical activity and selfefficacy in relation to exercise and improved management of the participants' everyday lives. ${ }^{30}$ However, Cederbom et al suggested that the intervention should be evaluated using a larger sample, with the goal of producing evidence-based guidelines for physical therapists. ${ }^{30}$

Thus, the primary aim of the planned study is to evaluate the effects of an individually tailored integrated BMPI on pain-related disability and physical performance by comparing the effects to standard care among older adults with chronic musculoskeletal pain who live alone at home and depend on formal care to manage their everyday lives. The second aim of the planned study is to evaluate the effect of the intervention on pain severity, pain-related beliefs, physical activity levels, falls efficacy, number of falls, and health-related quality of life (HRQoL).

\section{Methods/design Overview of study design}

The planned study is a pragmatic randomized controlled trial (RCT) $)^{31,32}$ with one intervention and one control group. The intervention is a 12 -week home-based program. The intervention consists of an individually tailored BMPI that is intended to decrease pain-related disability and pain-related 
beliefs (ie, fear of falling and pain catastrophizing) and improve physical performance and HRQoL, which could lead to an improved ability to perform everyday activities. The control group will be given standard care, which will include general advice about the benefits of physical activity in relation to pain problems. The sample will comprise $\sim 150$ participants who will be assessed at baseline and at 3 and 6 months after baseline.

A computer-generated, permuted-block randomization scheme with a 1:1 ratio between the intervention and control groups will be used, ${ }^{32}$ and the software program Stata 14 (StataCorp LLC, College Station, TX, USA) will be used to carry out the randomization. The randomization list will be prepared by a researcher who will not be involved in the remainder of the project. Sequentially numbered opaque sealed envelopes providing details regarding the group allocation will be prepared for each participant by a researcher who will not be involved in enrolling the participants. The randomization of each participant will be carried out after the baseline assessment, which means that the assessor at each baseline assessment will not know which group the participant will be placed in, and the assessors at the followup assessments will also be blind to the group allocation.

The study design follows the criteria set out in the CONSORT statement for reporting RCTs, ${ }^{32}$ and the protocol follows the Standard Protocol Items: Recommendation for Interventional Trials (SPIRIT) 2013 statement. ${ }^{33}$ The planned study is registered at ClinicalTrials.gov under the identifier NCT02953470. Enrollment into the study is to start in autumn 2017, and it is likely that it will be finished by the end of 2018. The intervention will be ongoing during the same time interval. Data collection will last for 6 months after enrollment of each participant. Thereafter, the authors will write up and publish peer-reviewed articles based on the results of the RCT.

\section{Study population}

The trial will be conducted in 5-15 districts in the municipality of Oslo in Norway. The study will include the districts that are nearest to the city center. These districts represent both high- and low-income populations with varying education levels. The inclusion criteria will be as follows:

- being aged $\geq 75$ years;

- living alone in ordinary housing;

- being dependent on home help services for individual care, housekeeping activities, and/or for medical needs but not being dependent on formal care (regarding ADL) as a result of a moderate or higher degree of cognitive impairment (as assessed based on the data in the municipal registry);

- having musculoskeletal pain for $\geq 3$ months;

- ability to walk independently indoors, with or without a walking aid;

- ability to understand and speak Norwegian;

- ability to fill in an activity diary or have contact with someone who can help to fill in the diary.

The exclusion criteria will be as follows:

- having ongoing physiotherapy treatment for injury/illness;

- receiving palliative treatment;

- experienced heart failure in the past 3 months.

The names and addresses of potential participants will be collected from a municipal registry that records the details of older adults who receive support from the municipality.

\section{Ethics approval and consent to participate}

The Regional Committee for Medical Research Ethics Norway has approved the planned study (reference number 2016/859). Each participant will receive oral and written information about the study, and they will be informed that their participation would be voluntary and that they would be able to withdraw from the study at any time. Before inclusion into the study, the participants will be asked for their oral and written informed consent.

\section{Intervention and control groups}

Both groups will receive general oral and written advice about physical activity during the first visit based on recommendations for physical activity for older adults aged $\geq 65$ years devised by the World Health Organization and American College of Sports Medicine. ${ }^{34,35}$ The participants will also receive information about the known benefits of physical activity in relation to the effects of activity on pain problems. ${ }^{36}$

\section{Individually tailored integrated behavioral medicine in physical therapy intervention}

The design of the BMPI is characterized by systematic consideration of the participants' physical and psychological conditions and the social and physical environmental factors related to the treatment goal. To identify each participant's treatment goal, a functional behavioral analysis (FBA) will be carried out by a physical therapist - together with the participant - in order identify either a problematic everyday activity or an activ- 
ity that it is highly important to maintain. The FBA includes identifying the physical, psychological, and environmental factors that inhibit and/or promote the participant's ability to perform the activity. The chosen activity is known as the "goal behavior," and it is designed to be "SMART," which means it is a specific measurable activity based on a limited time. FBAs are based mainly on the concept of "operant conditioning," the concept of how behavior is controlled by the consequences of the behavior.' With an FBA as a base, an individual's skills that require improvement can be identified, whether these are physical, behavioral, cognitive, or social skills. ${ }^{37,38}$ Examples of goal behavior for the target population are as follows: to climb stairs safely, to be able to stand without physical support when doing the washing up, and to maintain the ability to walk indoors without a walking aid. The FBA also involves identifying an individual's thoughts about the short- and long-term consequences of not changing their behavior. The FBAs result in the formulation of a hypothesis regarding the relationships between the physical, psychological, and environmental factors that influence the goal behavior.

A key factor in reaching the treatment goal is to increase the participant's physical exercise levels, in line with a statement from the American Geriatric Society that specified that interventions concerning physical exercise should be part of the care provided to older adults with chronic pain. ${ }^{36}$ The type of exercise that will be used to improve the physical issues experienced by each participant is functional exercise. The number and intensity of exercise sessions will be based on how much the participant is able and wants to do, and the number and intensity will increase progressively according to changes in the participant's performance and health status. ${ }^{39}$

Another key factor of the intervention is to enhance and improve self-efficacy in relation to the goal behavior. ${ }^{38} \mathrm{As}$ described by Albert Bandura, "self-efficacy" is a central concept in social cognitive theory that refers to the strength of one's beliefs in one's ability to complete tasks (using specific behavior) in order to achieve specific goals. ${ }^{40}$ Self-efficacy is an important factor if an individual is to achieve some form of behavioral change, for example, an increase in his or her physical activity levels. Self-efficacy beliefs also play an important role in embracing a new behavior, generalizing the new behavior to other situations, and maintaining the altered behavior over time. ${ }^{41}$ A low degree of self-efficacy also seems to be associated with chronic pain, pain-related disability, and depression among older adults living with pain. ${ }^{42}$ The evidence regarding the role that self-efficacy plays in various contexts is extensive, and it includes evidence regarding self-efficacy's positive effects on physical performance, the management of the psychological aspects of pain, and pain-related disability. ${ }^{25}$
During the intervention period, each participant will receive visits from a physical therapist once a week for weeks $1-8$ and 12. In week 10, the participant will receive support during a single telephone call. The content of the visits will be as follows: sessions 1-2: identification of a problematic or an important everyday activity that has to be maintained, and provision of advice about physical activity and information about how to fill in the activity diary. Performing an FBA, including goal setting and discussing how to carry out the physical activity and the intensity of the activity. Sessions 3-5: training in basic physical and psychological skills, for example, strengthening self-efficacy in relation to the goal behavior and decreasing fear of falling, catastrophizing thoughts, or fear of movement with regard to the goal behavior. Sessions 6-9: training in how to apply basic skills effectively to achieve the goal behavior, application of basic and more complex skills to other behaviors, and starting with secondary activity goals. Discussion of strategies for maintaining the new behavior. Discussion of problem-solving strategies, ie, how the participant could prevent and address new problems related to the goal behavior (in relation to being physically active) that may arise.

\section{Control group}

The control group will receive standard care, which will comprise one visit from a physical therapist who will give general oral and written advice regarding physical activity. Furthermore, the control group will receive telephone calls once a week from a physiotherapist during weeks 1-8 and 10 , in the same order as the individuals in the intervention group will receive visits from a physiotherapist. The telephone call will consist of a reminder to be physically active in accordance with the advice given during the first visit. No advice on specific exercises will be given.

\section{Training of the intervention providers}

The project leader (who holds a $\mathrm{PhD}$ ) and is a physical therapist (who is thoroughly trained and has comprehensive knowledge and competence regarding BMPIs), and research assistants (who are all physical therapists) will be involved in the recruitment and data collection. The assistants will receive education about the theoretical framework behind the study and the BMPI, and training in performing the data collection procedures, as well as delivering the intervention. To ensure that the data collection is performed consistently, the project leader will carry out a single baseline assessment with each assistant at the beginning. The assistants will receive regular supervision by the project leader during the intervention and data collection period so that they can discuss any issues related to the project. 


\section{Adherence}

The participants in the intervention group will be asked to carry out general physical activity on $\geq 5$ days per week, to carry out exercises related to their goal behavior on $\geq 5$ days per week, and to attempt to carry out the goal behavior on $\geq 1$ day per week. Adherence will be checked using the participants' activity diaries.

\section{Adverse events}

Adverse events will be recorded in the following four categories: falls, cardiovascular events, musculoskeletal injuries, and use of health care facilities. Issues related to the feasibility of the study procedure and the intervention protocol will be recorded in field notes during the intervention. The field notes will include comments about the time needed to collect the data, adherence to the study procedure and intervention protocol, and issues that arise in relation to the study procedure and intervention protocol.

\section{Outcomes}

Chronic pain is a multidimensional experience and, therefore, multiple outcomes will be used to detect potential changes. The participants will be assessed at baseline and at 3 months (follow-up 1) and 6 months (follow-up 2) after baseline. Table 1 provides an overview of the outcomes and assessment timepoints.
At baseline, the following demographic data will be collected: age (years), gender, marital status, amount of help from home help services and/or home health care services, use of walking aids indoors and outdoors, ability to get outdoors, number of self-reported medical diagnoses, self-reported use of prescribed and non-prescribed pain medication, number of years with pain, number of falls last year, and whether the participant feels as physically active as they wish to be.

The assessments will be interview administrated, and they will be conducted in a standardized way, which means that all the assessments for each participant will be carried out in the same order.

\section{Primary outcomes}

The primary outcomes will be pain-related disability and physical performance. Pain-related disability will be measured using the pain-related interference scale in the short Norwegian version of the brief pain inventory (BPI). ${ }^{43,44}$ The BPI is a self-report pain assessment tool that requires the respondent to report their pain severity and disability related to seven functions. ${ }^{44}$ The pain-related interference scale involved seven items that assess the interference of the respondent's pain with general activity, mood, walking ability, normal work, relationships with other people, sleep, and enjoyment of life. The response alternatives are based on numerical rating scales

Table I Summary of outcomes

\begin{tabular}{|c|c|c|c|c|}
\hline \multirow[t]{2}{*}{ Outcome } & \multirow[t]{2}{*}{ Data collection measurements } & \multicolumn{3}{|c|}{ Timepoints } \\
\hline & & Baseline & $\begin{array}{l}\text { Follow-up I } \\
\text { (3 months) }\end{array}$ & $\begin{array}{l}\text { Follow-up } 2 \\
\text { (6 months) }\end{array}$ \\
\hline \multicolumn{5}{|l|}{ Primary outcomes } \\
\hline Pain-related disability & $\begin{array}{l}\text { Pain-related interference scale in the brief pain } \\
\text { inventory }\end{array}$ & $\mathrm{X}$ & $\mathrm{X}$ & $\mathrm{X}$ \\
\hline Physical performance & Short physical performance battery & $\mathrm{X}$ & $\mathrm{X}$ & $\mathrm{X}$ \\
\hline \multicolumn{5}{|l|}{ Secondary outcomes } \\
\hline Pain severity & Pain severity scale in the brief pain inventory & $\mathrm{X}$ & $\mathrm{X}$ & $\mathrm{X}$ \\
\hline Pain catastrophizing & $\begin{array}{l}\text { Two items from the coping strategies } \\
\text { questionnaire }\end{array}$ & $X$ & $\mathrm{X}$ & $X$ \\
\hline Falls-efficacy & Falls efficacy scale-international & $\mathrm{X}$ & $\mathrm{X}$ & $\mathrm{X}$ \\
\hline Level of physical activity & 6-point scale & $\mathrm{X}$ & $\mathrm{X}$ & $\mathrm{X}$ \\
\hline Health-related quality of life & I2-item Short-Form Health Survey & $\mathrm{X}$ & $\mathrm{X}$ & $\mathrm{X}$ \\
\hline Assessment of perceived result & Consumer questions & & $\mathrm{X}$ & $\mathrm{X}$ \\
\hline $\begin{array}{l}\text { Self-efficacy in relation to goal } \\
\text { behavior (for the intervention } \\
\text { group) }\end{array}$ & 4-point scale & $X$ & $X$ & \\
\hline $\begin{array}{l}\text { Reached goal behavior (for } \\
\text { intervention group) }\end{array}$ & Yes/no question & & $X$ & \\
\hline Number of falls & $\begin{array}{l}\text { Question on how many falls occurred last year, } \\
\text { during the intervention period, and between } \\
\text { follow-up I and follow-up } 2\end{array}$ & $X$ & $\mathrm{X}$ & $X$ \\
\hline
\end{tabular}


from 0 to 10 , where 0 represents "does not interfere" and 10 represents "interferes completely." The BPI has been validated for use in older adults with noncancer pain. ${ }^{45}$

Physical function will be measured using the Norwegian version of the Short Physical Performance Battery (SPPB). ${ }^{46,47}$ The SPPB evaluates balance, mobility, and muscle strength by examining an individual's ability to stand with their feet side by side, in a semi-tandem position, and in a tandem position, the time taken to walk $3 \mathrm{~m}$, and the time taken to rise from a chair and return to a seated position five times. Each of the three tests is given a score, based on performance, between 0 and 4 , which sum to a maximum score of 12 for the individuals who performed the three activities at the highest level of performance. The SPPB has been shown to be a reliable and valid measure of lower extremity performance, and it is therefore suitable for evaluating physical performance. ${ }^{46}$

\section{Secondary outcomes}

The study will include several secondary outcomes. First, the severity of the pain will be measured by using the pain severity items in the BPI (see the "Primary outcomes" section regarding the BPI). The BPI pain severity scale involves measuring the highest, lowest, and mean pain intensity related to four items (usually with respect to the current intensity and the intensity during the past 24 hours or past week, and intensity now). The response for each item is bounded by the words "no pain" and "worst pain you can imagine."

Pain catastrophizing will be measured by using the following two items from the Norwegian version of the Coping Strategies Questionnaire: "It is terrible and I feel it is never going to get any better" and "I feel I can't stand it anymore." 48,49 The response scale ranges from 0 to 6 , where 0 is defined as "never think that way" and 6 is defined as "always think that way." The two-item scale has been shown to be both valid and reliable for use in adults who report having chronic pain. ${ }^{48}$

Falls efficacy will be assessed using the Norwegian version of the Falls Efficacy Scale-International (FES-I). ${ }^{50,51}$ The FES-I assesses the level of concern about falling for 16 activities of daily living, with each assessment being based on a 4-point scale. Scores range from 16 to 64, with higher scores indicating greater concern about falling. The FES-I has been shown to be both a valid and reliable measure for use in older people. ${ }^{30,51}$

The level of physical activity, including housekeeping activities, will be measured by using a 6-point scale. ${ }^{52}$ The response scale ranges from levels 1 to 6 , where level 1 is defined as "hardly any physical activity" and level 6 is defined as "hard exercise several times a week." The scale has been shown to be valid for use in older adults. ${ }^{53}$

HRQoL will be measured by using the Norwegian version of the 12-item Short-Form Health Survey (SF-12), which is a shortened version of the 36-item Short-Form Health Survey (SF-36). The SF-12 covers 8-HRQoL domains: 1) physical functioning (2 items); 2) role-physical, which is role limitations due to physical problems (2 items); 3 ) bodily pain (1 item); 4) general health (1 item); 5) vitality (1 item); 6) social functioning (1 item); 7) role-emotional, which is role limitations due to emotional problems (2 items); and 8) mental health (2 items). Based on these domains, summary scores for the physical component (domains 1-4) and mental component (domains 5-8) will be computed. ${ }^{54,55}$ TheSF-12 has been shown to be valid for use in older adults. ${ }^{56}$

To assess the participants' perception of the results of the intervention, the following "consumer questions" will be asked:

- how is your physical activity level now, after participation in the study?;

- overall, how do you manage your everyday life compared with how it was before the intervention?;

- are you using what you learned during the intervention to manage your physical issues or prevent further physical issues?;

- do you take any pain medication?;

- have you been in contact with a physical therapist during the intervention period or since you finished participation in the study? ${ }^{30}$

In addition, at follow-up 2, the following question will be asked:

- do you still exercise or are you being physically active?.

Regarding falls, the participants will be asked if they had any falls during the intervention period or between followup 1 and follow-up 2. In addition, for the participants in the intervention group, whether or not the goal behavior has been reached and the participants' self-efficacy in relation to the goal behavior will be assessed.

\section{Sample size calculation and statistical analyses}

Power calculation was carried out for both primary outcomes, but there was no difference in the sample sizes calculated. The sample size calculation was based on a small meaningful change in the BPI and SPPB. For the BPI, a small meaningful change has been defined as a mean (SD) of $1(0.5)$ points. $^{57}$ For the SPPB, a small meaningful change has been defined as 
a mean (SD) of 0.5 (1.48) points. ${ }^{58}$ To detect this effect when the power is $80 \%$ and the significance level (alpha) is 0.05 , a sample size of 138 participants (69 in each group) would be needed (based on the size of a small meaningful change for the BPI or SPPB). We will include 150 participants in order to compensate for potential dropouts.

The data will all be analyzed and presented in accordance with the CONSORT statement for reporting parallel-group RCTs. ${ }^{32}$ Differences between groups will be analyzed with independent samples $t$-tests and differences between groups will be analyzed with paired samples $t$-tests. Intention-to-treat approach and missing data will be dealt with by carrying the last known values forward. Effect sizes will be calculated. If possible, further analyses will be carried out to examine relationships and/or correlations. ${ }^{59}$ The significance level will be set at $P<0.05$.

\section{Discussion}

It is believed that the planned study will be the first RCT to evaluate the effect of an evidence-based BMPI for older adults with chronic musculoskeletal pain. Should the intervention be shown to be effective, it could have positive effects both for older adults with chronic pain and for the practitioners who work with this population, and the study could make an important contribution to the evidence base.

We expect that the intervention described in this protocol will reduce pain-related disability and beliefs and increase physical performance and physical activity levels compared to the provision of basic advice about physical activity and reminder by telephone to follow the advice. The intervention may maintain or improve each participant's ability to perform everyday activities and their quality of life. In turn, this may allow the participants to live at home for longer. The results of the intervention will have the potential to promote evidence-based decision making among health practitioners and patients. The intervention will be home-based, as earlier studies have found this to be a successful approach for improving both participation and adherence in interventional studies with older people..$^{30,60}$

Regarding physical activity, we know that older adults with chronic pain are less physically active compared to older adults without pain. In addition, older people who live alone are less physically active than those who live with someone, and older women are less physically active than older men. ${ }^{26,38,61}$ A low level of physical activity can endanger health, independence, and quality of life. ${ }^{61}$ To promote increased physical activity levels and to increase the positive effects that physical activity brings about, the planned study will have a special focus on physical activity.
The intervention has been designed to enhance the participants' desire to be physically active for $\geq 30 \mathrm{~min} /$ day, in line with the World Health Organization and American College of Sports Medicine recommendations. ${ }^{34}$

Individually tailored interventions can be challenging because of the variety of the interventions, but they may also be the most effective interventions, and they are recommended for use when carrying out interventions among older adults. ${ }^{62} \mathrm{~A}$ BMPI is a complex intervention, and those involved in implementing a BMPI require a comprehensive education regarding its multidimensional perspectives and the steps needed for the BMPI to succeed. ${ }^{26,38}$ The assistants who are to be involved in the study will be fully trained and they will have regular supervision by the project leader, who has the knowledge and competence to carry out this type of intervention.

From a scientific perspective, regardless of whether the results of the study are positive or negative, the results will contribute to knowledge about the effects of BMPIs in the target population, which in itself will be very interesting. If the results are positive, they might become the basis for evidence-based guidelines for managing chronic pain in the target population.

Before implementing an RCT, it is important to consider issues related to the quality of the study, including internal and external validity. To increase the internal and external validity, a systematic approach (based on guidance on designing and evaluating the quality of RCTs) has been followed. ${ }^{32}$

To increase the potential of the study to contribute to evidence-based guidelines for health practitioners, the study has a pragmatic design. The pragmatic design will not only allow us to obtain valuable data with a high degree of external validity but also lay the foundation for producing potentially clinically applicable findings. ${ }^{31}$ Community-dwelling older adults were selected as the target population in order to assess the intervention using realistic conditions. The inclusion criteria were designed to encompass a representative group of participants. ${ }^{63}$ In addition, the high-risk nature of the target population might enhance the adverse event detection rates. The intervention has been well described and the outcome measures were selected carefully so that they will be easy for the assessors to use and the participants to understand.

The outcome measures have been used previously among community-dwelling older adults with chronic musculoskeletal pain, and they have been shown to be both reliable and valid in the target population. To maximize the test-retest reliability, the assessors will be experienced physical therapists who will be comprehensively trained in how to carry out assessments before enrollment begins. Due to its large sample size and the strong theoretical basis of the 
intervention, the planned study has the potential to generate new knowledge that may improve the design of future interventions for community-dwelling older adults with chronic musculoskeletal pain.

\section{Acknowledgments}

The authors thank Professor Are Pripp for help with the randomization plan and the calculation of the sample size for the study.

The study is funded by the Norwegian Physical Therapist Association. The funders have had no role in the study design, data collection and analysis, decision to publish, or preparation of the manuscript.

\section{Author contributions}

$\mathrm{SC}$ conceived of the study. SC, ED, and $\mathrm{AB}$ participated in the design of the study. SC, together with a statistician, calculated the required sample size, and $\mathrm{SC}, \mathrm{ED}$, and $\mathrm{AB}$ developed the plan for the statistical analysis of the trial outcomes. SC will be responsible for the trial management, recruitment, and data collection. SC drafted the manuscript with contributions from all the other authors. All the authors have read and approved the final manuscript.

\section{Disclosure}

The authors report no conflicts of interest in this work.

\section{References}

1. Socialstyrelsen. Vård och omsorg om äldre. Lägesrapport 2008. Insatser och stöd till personer med funktionsnedsättning. Individochomsorgomäldre. The Swedish National Board of Health and Welfare. Care and social services for the elderly. Progress Report; 2008.

2. Sørbye LW, Garms-Homolová V, Henrard J-C, et al. Shaping home care in Europe: the contribution of the Aged in Home Care project. Maturitas. 2009;62(3):235-242.

3. Wiles JL, Leibing A, Guberman N, Reeve J, Allen RE. The meaning of "ageing in place" to older people. Gerontologist. 2011;52(3):357-366.

4. Tang F, Lee Y. Home- and community-based services utilization and aging in place. Home Health Care Serv Q. 2010;29(3):138-154.

5. Oberski IM, Carter DE, Gray M, Ross J. The community gerontological nurse: themes from a needs analysis. J Advanced Nurs. 1999;29(2):454-462.

6. Sjölund B-M. Physical functioning in old age. Temporal trends and geographical variations in Sweden. Karolinska Institutet; 2014. Available from https://openarchive.ki.se/xmlui/handle/10616/41968. Accessed March 31, 2017.

7. Gibson SJ, Lussier D. Prevalence and relevance of pain in older persons. Pain Med. 2012;13(Suppl 2):S23-S26.

8. Larsson Å, Haglund L, Hagberg J-E. Doing everyday life-experiences of the oldest old. Scandinavian J Occupational Ther. 2009;16(2):99-109.

9. Covinsky KE, Palmer RM, Fortinsky RH, et al. Loss of independence in activities of daily living in older adults hospitalized with medical illnesses: increased vulnerability with age. JAGS. 2003;51(4):451-458.

10. Fortinsky RH, Covinsky KE, Palmer RM, Landefeld CS. Effects of functional status changes before and during hospitalization on nursing home admission of older adults. J Gerontol A Biol Sci Med Sci. 1999;54(10):M521-M526.
11. Covinsky KE, Justice AC, Rosenthal GE, Palmer RM, Landefeld CS. Measuring prognosis and case mix in hospitalized elders. J Gen Intern Med. 1997;12(4):203-208.

12. Henderson A, Scott R, Kay D. The elderly who live alone: their mental health and social relationships. Aust NZ Psychiatry. 1986;20(2):202-209.

13. Kharicha K, Iliffe S, Harari D, Swift C, Gillmann G, Stuck AE. Health risk appraisal in older people 1: are older people living alone an 'at-risk' group? Br J Gen Pract. 2007;57(537):271-276.

14. Karp A, Agahi N, Lennartsson C, Lagergren M, Wånell S-E. Healthy aging. A review of the literature between 2005-2012 about promoting healthy aging on the individual level. Stockholm Gerontology Research Center. 2013.

15. Lennartsson C, Agahi N, Wånell S-E. Äldrepersonershälsaochlivssituation. Läget i Stockholms läns och utmaningar för det fortsatta hälsoarbetet. Older Peoples health and life situation. The situation iSthockholm and challenges for the continuing health promotion work. Rapporter/Stiftelsen Stockholms läns Äldrecentrum. 2012:8.

16. Bradbeer M, Helme RD, Yong HH, Kendig HL, Gibson SJ. Widowhood and other demographic associations of pain in independent older people. Clin J Pain. 2003;19(4):247-254.

17. Soldato M, Liperoti R, Landi F, et al. Non malignant daily pain and risk of disability among older adults in home care in Europe. Pain. 2007; 129(3):304-310.

18. Eggermont LH, Leveille SG, Shi L, et al. Pain characteristics associated with the onset of disability in older adults: the maintenance of balance, independent living, intellect, and zest in the elderly Boston study. $\mathrm{J} \mathrm{Am}$ Geriatr Soc. 2014;62(6):1007-1016.

19. Martin R, Hadjistavropoulos T, McCreary D. Fear of pain and fear of falling among younger and older adults with musculoskeletal pain conditions. Pain Res Manag. 2005;10(4):211-219.

20. Lundberg MKE. A psychometric evaluation of the Tampa Scale for Kinesiophobia - from a physiotherapeutic perspective. Physiother Theory Pract. 2004;20(2):121.

21. Leeuw M, Goossens ME, Linton SJ, Crombez G, Boersma K, Vlaeyen JW. The fear-avoidance model of musculoskeletal pain: current state of scientific evidence. J Behav Med. 2007;30(1):77-94.

22. Cederbom S, Söderlund A, Denison E, von HeidekenWågert P. Chronic pain among older women living alone. A study focusing on disability and morale. Eur J Physiother. 2014;16:139-150.

23. Gillespie LD, Robertson MC, Gillespie WJ, et al. Interventions for preventing falls in older people living in the community. Cochrane Database Syst Rev. 2012;9(11):CD007146.

24. Stubbs B, West E, Patchay S, Schofield P. Is there a relationship between pain and psychological concerns related to falling in community dwelling older adults? A systematic review. Disabil Rehab. 2014;36: 1931-1942.

25. Keefe FJ, Rumble ME, Scipio CD, Giordano LA, Perri LM. Psychological aspects of persistent pain: current state of the science. J Pain. 2004;5(4):195-211.

26. Cederbom S. Ability in Everyday Activities and Morale among Older Women with Chronic Musculoskeletal Pain Living Alone [doctoral thesis]. Stockholm: KarolinskaInstitutet; 2014.

27. Swedish Council of Health Technology Assessments. Rehabilitation of Patients with Chronic Pain Conditions. 2010. SBU-report nr 198. 2010. Available from http://www.sbu.se/globalassets/publikationer/ content1/1/smartrehab_eng_smf_110518.pdf. Accessed March 31, 2017.

28. Emilson C, Demmelmaier I, Bergman S, Lindberg P, Denison E, Åsenlöf P. A 10-year follow-up of tailored behavioural treatment and exercisebased physiotherapy for persistent musculoskeletal pain. Clinical Rehab. 2016:0269215516639356.

29. Söderlund A, Lagerlöf $H$. An individually tailored behavioral medicine treatment in physical therapy for tension-type headache - two experimental case studies. J Pain Res. 2016;23(9)1089-1099.

30. Cederbom S, Rydwik E, Dension E, Söderlund A, Frändin K, Von HeidekenWagert P. A behavioral medicine intervention for older women living alone with chronic pain - a feasibility study. Clin Intervent Aging. 2014;9:1383-1397. 
31. Patsopoulos NA. A pragmatic view on pragmatic trials. Dialogues Clin Neurosci. 2011;13(2):217-224.

32. Schulz KF, Altman DG, Moher D. CONSORT 2010 statement: updated guidelines for reporting parallel group randomised trials. $B M C \mathrm{Med}$. 2010;8(1):1.

33. Chan A-W, Tetzlaff JM, Gøtzsche PC, et al. SPIRIT 2013 explanation and elaboration: guidance for protocols of clinical trials. $B M J$. 2013;346:e7586.

34. World Health Organization. [webpage on the Internet]. Global recommendations on physical activity for health. 2010. Available from http:// www.who.int/dietphysicalactivity/factsheet_recommendations/en/. Accessed March 31, 2017.

35. Nelson ME, Rejeski W, Blair SN, et al. Physical activity and public health in older adults: recommendation from the American College of Sports Medicine and the American Heart Association. Med Sci Sports Exercise. 2007;39(8):1435-1445.

36. Exercise prescription for older adults with osteoarthritis pain: consensus practice recommendations. A supplement to the AGS Clinical Practice Guidelines on the management of chronic pain in older adults. $J \mathrm{Am}$ Geriatr Soc. 2001;49(6):808-823.

37. Åsenlöf P, Denison E, Lindberg P. Individually tailored treatment targeting activity, motor behavior, and cognition reduces pain-related disability: a randomized controlled trial in patients with musculoskeletal pain. J Pain. 2005;6(9):588-603.

38. Denison E, Åsenlöf P. Behavioural Medicine Applications in Physiotherapy. Lund: StudentlitteraturAB; 2012.

39. Littbrand H, Rosendahl E, Lindelöf N, Lundin-Olsson L, Gustafson Y, Nyberg L. A high-intensity functional weight-bearing exercise program for older people dependent in activities of daily living and living in residential care facilities: evaluation of the applicability with focus on cognitive function. Physical Ther. 2006;86(4):489-498.

40. Bandura A. Self-Efficacy: The Exercise of Control. New York: Freeman; 1997.

41. Bandura A. Self-efficacy: toward a unifying theory of behavioral change. Psychol Rev. 1977;84(2):191-215.

42. Turner JA, Ersek M, Kemp C. Self-efficacy for managing pain is associated with disability, depression, and pain coping among retirement community residents with chronic pain. J Pain. 2005;6(7):471.

43. Klepstad P, Loge JH, Borchgrevink PC, Mendoza TR, Cleeland CS, Kaasa $\mathrm{S}$. The Norwegian brief pain inventory questionnaire: translation and validation in cancer pain patients. J Pain Symptom Manage. 2002; 24(5):517-525.

44. Cleeland CS, Ryan K. The brief pain inventory. Pain Research Group. 1991. Available from http://www.rygforskning.dk/sites/default/files/ files/skemaer/BPI_UserGuide.pdf. Accessed March 31, 2017.

45. Bann C, Dodd SL, Schein J, Mendoza TR, Cleeland CS. Validity of the brief pain inventory for use in documenting the outcomes of patients with noncancer pain. Clin J Pain. 2004;20(5):309-318.

46. Guralnik JM, Ferrucci L, Pieper CF, et al. Lower extremity function and subsequent disability consistency across studies, predictive models, and value of gait speed alone compared with the short physical performance battery. J Gerontol A Biol Sci Med Sci. 2000;55(4):M221-M231.

47. Prestmo A, Hagen G, Sletvold O, et al. Comprehensive geriatric care for patients with hip fractures: a prospective, randomised, controlled trial. Lancet. 2015;385(9978):1623-1633.
48. Jensen MP, Keefe FJ, Lefebvre JC, Romano JM, Turner JA. Oneand two-item measures of pain beliefs and coping strategies. Pain $2003 ; 104(3): 453-469$

49. Utne I, Miaskowski C, Bjordal K, Paul SM, Jakobsen G, Rustøen T. Differences in the use of pain coping strategies between oncology in patients with mild vs. moderate to severe pain. J Pain Symptom Manage. 2009;38(5):717-726.

50. Helbostad JL, Taraldsen K, Granbo R, Yardley L, Todd CJ, Sletvold O. Validation of the Falls Efficacy Scale-International in fall-prone older persons. Age Ageing. 2010;39(2):259.

51. Kempen GI, Todd CJ, Van Haastregt JC, et al. Cross-cultural validation of the Falls Efficacy Scale International (FES-I) in older people: results from Germany, the Netherlands and the UK were satisfactory. Disabil Rehab. 2007;29(2):155-162.

52. Frändin K, Grimby G. Assessment of physical activity, fitness and performance in 76-year-olds. Scand J Med Sci Sports. 1994;4(1): 41-46.

53. Mattiasson-Nilo I, Sonn U, Johannesson K, Gosman-Hedström G, Persson G, Grimby G. Domestic activities and walking in the elderly: evaluation from a 30-hour heart rate recording. Aging. 1990;2(2):191.

54. Ware JE Jr, Kosinski M, Keller SD. A 12-Item Short-Form Health Survey: construction of scales and preliminary tests of reliability and validity. Med Care. 1996;34(3):220-233.

55. Loge JH, Kaasa S, Hjermstad MJ, Kvien TK. Translation and performance of the Norwegian SF-36 Health Survey in patients with rheumatoid arthritis. I. Data quality, scaling assumptions, reliability, and construct validity. J Clin Epidemiol. 1998;51(11):1069-1076.

56. Jakobsson U. Using the 12-item Short Form health survey (SF-12) to measure quality of life among older people. Aging Clin Exp Res. 2007; 19(6):457-464.

57. Dworkin RH, Turk DC, Wyrwich KW, et al. Interpreting the clinical importance of treatment outcomes in chronic pain clinical trials: IMMPACT Recommendations. J Pain. 2008;9(2):105-121.

58. Perera S, Mody SH, Woodman RC, Studenski SA. Meaningful change and responsiveness in common physical performance measures in older adults. JAGS. 2006;54(5):743-749.

59. Altman DG. Practical Statistics for Medical Research. CRC Press, USA; 1990.

60. Arkkukangas M, Johnson ST, Hellström K, Söderlund A, Eriksson S, Johansson A-C. A feasibility study of a randomised controlled trial comparing fall prevention using exercise with or without the support of motivational interviewing. Prevent Med Rep. 2015;2: $134-140$.

61. Stubbs B, Binnekade TT, Soundy A, Schofield P, Huijnen IP, Eggermont LH. Are older adults with chronic musculoskeletal pain less active than older adults without pain? A systematic review and meta-analysis. Pain Med. 2013;14(9):1316-1331.

62. Conradsson M, Littbrand H, Lindelof N, Gustafson Y, Rosendahl E. Effects of a high-intensity functional exercise programme on depressive symptoms and psychological well-being among older people living in residential care facilities: a cluster-randomized controlled trial. Aging Ment Health. 2010;14(5):565-576.

63. McMurdo ME, Roberts $\mathrm{H}$, Parker $\mathrm{S}$, et al. Improving recruitment of older people to research through good practice. Age Ageing. 2011;40(6): 659-665.

\section{Journal of Pain Research}

\section{Publish your work in this journal}

The Journal of Pain Research is an international, peer reviewed, open access, online journal that welcomes laboratory and clinical findings in the fields of pain research and the prevention and management of pain. Original research, reviews, symposium reports, hypothesis formation and commentaries are all considered for publication
The manuscript management system is completely online and includes a very quick and fair peer-review system, which is all easy to use. Visit http://www.dovepress.com/testimonials.php to read real quotes from published authors. 